\title{
Fosfomycin Susceptibility in Multidrug- Resistant Enterobacteriaceae Species and Vancomycin-Resistant Enterococci Urinary Isolates
}

\author{
Linda B Ou and Lynn Nadeau
}

\begin{abstract}
Background: Broad-spectrum antibiotics are often used to treat urinary tract infections (UTIs) due to drug-resistant species of Enterobacteriaceae and Enterococcus (e.g., organisms producing extended-spectrum ß-lactamase [ESBL] or AmpC ß-lactamase, as well as vancomycin-resistant enterococci [VRE]). However, this type of therapy can promote selection of resistant organisms and may necessitate venous access. Fosfomycin is an orally administered, single-dose antibiotic for the treatment of uncomplicated UTI. Little is known about its microbiologic activity against urinary isolates, including in southwestern Ontario, since fosfomycin susceptibility testing is not routinely performed.
\end{abstract}

Objective: To explore a cost-effective alternative for the treatment of lower UTIs caused by multidrug-resistant Enterobacteriaceae and VRE organisms resistant to usual first-line therapies by determining fosfomycin susceptibility rates.

Methods: Urinary isolates were collected prospectively from November 2015 to April 2016 at 3 hospitals in southwestern Ontario. Susceptibility testing was completed according to guidelines of the Clinical and Laboratory Standards Institute, with interpretation by zone of inhibition (as diameter in millimetres). Patients 18 years of age or older with isolation of multidrug-resistant Enterobacteriaceae or VRE were eligible for inclusion. Urinary isolates from these patients were subjected to susceptibility testing. The primary outcome was the rate of fosfomycin susceptibility of these isolates.

Results: A total of 137 urinary isolates were tested: 106 positive for ESBLor AmpC B-lactamase-producing Enterobacteriaceae (95 Escherichia coli, 11 Klebsiella spp.) and 31 positive for vancomycin-resistant Enterococcus faecium. Susceptibility rates for ESBL- and AmpC B-lactamase-producing E. coli were $100 \%$ for ertapenem, $96 \%$ for fosfomycin, $83 \%$ for nitrofurantoin, $72 \%$ for gentamicin, $56 \%$ for trimethoprim-sulfamethoxazole, and $14 \%$ for ciprofloxacin. Susceptibility rates of vancomycin-resistant E. faecium urinary isolates were $100 \%$ for linezolid, $81 \%$ for fosfomycin, $68 \%$ for tetracycline, $6 \%$ for ampicillin, 3\% for penicillin, and $0 \%$ for both nitrofurantoin and ciprofloxacin.

Conclusion: Given susceptibility rates at the study institutions, fosfomycin was deemed the most reliable oral option for the treatment of lower UTI in patients with suspected or documented multidrug-resistant uropathogens.

\section{RÉSUMÉ}

Contexte : Les antibiotiques à large spectre sont souvent employés pour traiter les infections urinaires causées par des espèces d'entérobactériacées et d'Enterococcus résistantes aux médicaments (par exemple, des organismes qui produisent des ß-lactamases à spectre étendu [BLSE] ou des ß-lactamases AmpC de même que des entérocoques résistants à la vancomycine $[E R V])$. Or, ce type de traitement peut favoriser la sélection d'organismes résistants et peut nécessiter un accès veineux. La fosfomycine est un antibiotique oral à dose unique servant au traitement d'infections urinaires non compliquées. On connaît peu de choses sur son activité microbiologique contre les isolats urinaires, en l'occurrence dans le sudouest de l'Ontario, car on ne teste pas systématiquement la fosfomycine dans les antibiogrammes.

Objectif : Chercher une solution ayant un bon rapport coût-efficacité pour le traitement des infections urinaires basses causées par des espèces d'entérobactériacées multirésistantes aux antibiotiques et des ERV qui ne répondent pas aux traitements de première intention normalement utilisés en déterminant les degrés de sensibilité à l'égard de la fosfomycine.

Méthodes : Des isolats urinaires ont été recueillis de façon prospective entre novembre 2015 et avril 2016 dans trois hôpitaux du sud-ouest de l'Ontario. Des antibiogrammes ont été réalisés selon les lignes directrices du Clinical and Laboratory Standards Institute, et l'interprétation était fondée sur la zone d'inhibition (soit le diamètre en millimètres). Les patients de 18 ans et plus chez qui on avait isolé des entérobactériacées ou des ERV multirésistants aux antibiotiques étaient admissibles à l'étude. Les isolats urinaires provenant de ces patients étaient soumis à un antibiogramme. Le principal paramètre d'évaluation était le taux de sensibilité à la fosfomycine des isolats urinaires.

Résultats : Au total, 137 isolats urinaires ont été testés; 106 étaient positifs pour des espèces d'entérobactériacées produisant des BLSE ou des ßlactamases AmpC (95 Escherichia coli, 11 espèces de Klebsiella) et 31 étaient positifs pour l'Enterococcus faecium résistant à la vancomycine. Les taux de sensibilité d' $E$. coli produisant des BLSE et des B-lactamases AmpC étaient de $100 \%$ pour l'ertapénem, $96 \%$ pour la fosfomycine, $83 \%$ pour la nitrofurantoïne, $72 \%$ pour la gentamicine, $56 \%$ pour le co-trimoxazole et $14 \%$ pour la ciprofloxacine. Les taux de sensibilité pour les isolats urinaires d'Enterococcus faecium résistant à la vancomycine étaient de $100 \%$ pour le linézolide, $81 \%$ pour la fosfomycine, $68 \%$ 
Keywords: fosfomycin, urinary tract infection, resistant Enterobacteriaceae species, extended-spectrum ß-lactamase, vancomycin-resistant enterococci, Escherichia coli pour la tétracycline, $6 \%$ pour l'ampicilline, $3 \%$ pour la pénicilline et $0 \%$ pour la nitrofurantoïne et la ciprofloxacine.

Conclusion : En raison des taux de sensibilité obtenus aux établissements à l'étude, la fosfomycine a été jugée comme le médicament oral le plus fiable pour le traitement des infections urinaires basses chez les patients pour lesquels la présence d'uropathogènes multirésistants aux antibiotiques est soupçonnée ou connue.

Mots clés : fosfomycine, infection urinaire, espèces d'entérobactériacées résistantes, ß-lactamases à spectre étendu, entérocoques résistants à la vancomycine, Escherichia coli

\section{INTRODUCTION}

$\mathrm{F}$ osfomycin, a phosphoric acid derivative, exerts its bactericidal action by inhibiting peptidoglycan assembly, thus disrupting bacterial cell wall synthesis. It also reduces adherence of bacteria to uroepithelial cells. In Canada, fosfomycin is available only as an oral formulation, and its sole indication is acute, uncomplicated lower urinary tract infection (UTI) caused by susceptible Escherichia coli or Enterococcus faecalis in women 18 years of age and older. ${ }^{1}$ Off-label use of multiple-dose regimens of fosfomycin for the treatment of complicated lower UTI is also supported by literature. ${ }^{2-4}$ Fosfomycin has a broad spectrum of activity against many gram-negative bacteria and some gram-positive bacteria. In recent years, increasing antibiotic resistance, specifically involving E. coli and Klebsiella spp. producing AmpC ß-lactamase and extended-spectrum ß-lactamase (ESBL) and vancomycin-resistant enterococci (VRE), has renewed interest in exploring fosfomycin's activity against these organisms.

Enterobacteriaceae species producing AmpC B-lactamase and ESBL are able to hydrolyze antibiotics, such as penicillins and cephalosporins, thereby inactivating these antibiotics and potentially resulting in treatment failure. ${ }^{5}$ According to regional antibiograms, the rate of ESBL-producing bacteria in the Windsor-Essex area rose from $10 \%$ in 2013 to $14 \%$ in 2015 . Similarly, the Study for Monitoring Antimicrobial Resistance Trends (SMART), conducted in the United States and Canada, showed that rates of ESBL-producing $E$. coli in urinary isolates increased from $7.8 \%$ in 2010 to $18.3 \%$ in 2014 in the United States, and from $10.4 \%$ to $13.0 \%$ in Canada. ${ }^{6}$ Co-resistance to trimethoprimsulfamethoxazole, nitrofurantoin, and fluoroquinolones is common among these pathogens, despite these drugs being common first-line therapies for UTIs. ${ }^{7}$ With limited treatment options available, prescribers may resort to using one of the carbapenems, specifically ertapenem, to treat these resistant organisms, because of its convenient once-daily administration and narrower spectrum of activity within the carbapenem class.
Carbapenem therapy for the treatment of UTIs is not ideal, given that these broad-spectrum antibiotics are typically reserved for treating severe and potentially life-threatening infections. Increased use could promote selection of carbapenem-resistant organisms, leaving limited therapeutic options when broad-spectrum antimicrobial coverage is needed for highly resistant bacteria. Furthermore, carbapenems are available only as IV formulations, thus necessitating venous access and increasing costs. ${ }^{8-10}$

Enterococci commonly cause UTIs in hospitalized patients, with E. faecalis and Enterococcus faecium being the most frequently isolated species. Ampicillin and vancomycin are usually the drugs of choice for treating infections caused by enterococci. VRE organisms are, by definition, resistant to vancomycin and are often also resistant to ampicillin. Newer agents such as linezolid and daptomycin have been used to treat VRE infections, but their use is limited by cost, need for venous access, toxicity concerns, drug interactions, and potential development of resistance. ${ }^{11}$

Several studies have shown that fosfomycin has good in vitro and in vivo activity against both ESBL-producing organisms and VRE. A meta-analysis of randomized controlled trials comparing fosfomycin with typical antibiotic therapy for the treatment of cystitis showed no significant difference in clinical and microbiological outcomes. ${ }^{12}$ Fosfomycin has several distinct advantages. Rapid attainment of therapeutic concentrations in the bladder leads to high concentrations in the urine for 72 to $84 \mathrm{~h}$, which allows the drug to be given as a single oral dose to treat acute cystitis. ${ }^{1}$ It also has minimal propensity for cross-resistance, few adverse events, and decreased risk of allergy (due to its unique molecular structure). ${ }^{13}$

This study aimed to examine the utility of fosfomycin in the study region by determining the rate of fosfomycin susceptibility of multidrug-resistant Enterobacteriaceae and VRE urinary isolates. The frequency of major risk factors associated with resistant uropathogens was also obtained. If supported by susceptibility, the use of fosfomycin might decrease the use of broad-spectrum antibiotics, reduce the need for IV access, and provide overall cost savings. 


\section{METHODS}

\section{Study Design and Participants}

This prospective, observational, multicentre study was conducted from November 1, 2015, to April 30, 2016, at Windsor Regional Hospital (Ouellette and Metropolitan campuses), HotelDieu Grace Healthcare, and Leamington District Memorial Hospital. Approval for this study was granted for all 3 institutions by the Research Ethics Board at Windsor Regional Hospital. Included in this study were all urinary isolates, from patients 18 years of age or older, that tested positive for ESBL- or AmpC B-lactamase-producing E. coli or Klebsiella spp. or VRE. Repeat urine cultures from the same patient within 30 days were excluded.

\section{Data Collection}

Urine samples for culture were collected during routine patient care and were processed in the usual manner by Integrated Hospital Laboratories Service, Windsor-Essex. In addition to standard susceptibility testing, all cultures that yielded VRE or resistant Enterobacteriaceae species (ESBL- or AmpC B-lactamaseproducing E. coli or Klebsiella spp.) were tested for fosfomycin susceptibility. Antibiotic susceptibility testing for ESBL- and AmpC ß-lactamase-producing urinary pathogens involved ertapenem, nitrofurantoin, gentamicin, trimethoprimsulfamethoxazole, and ciprofloxacin. With respect to VRE isolated from urine, the susceptibility panel consisted of linezolid, tetracycline, ampicillin, penicillin, nitrofurantoin, and ciprofloxacin. In accordance with guidelines of the Clinical and Laboratory Standards Institute, each resistant isolate was tested for fosfomycin susceptibility by disk diffusion on agar media supplemented with glucose-6-phosphate. More specifically, after application of a bacterial inoculum to the agar plate, a $200-\mu g$ fosfomycin disk containing $50 \mu \mathrm{g}$ of glucose- 6 -phosphate was placed on the plate for 16 to $18 \mathrm{~h}$ at a mean temperature of $35^{\circ} \mathrm{C}$ (standard deviation $2^{\circ} \mathrm{C}$ ) in ambient air. Fosfomycin susceptibility was interpreted by zone of inhibition (as diameter in millimetres). Zones of inhibition with diameter $16 \mathrm{~mm}$ or greater are considered to represent susceptibility, zones of 13-15 mm diameter represent intermediate susceptibility, and zones of $12 \mathrm{~mm}$ or less represent resistance. ${ }^{14,15}$ Breakpoints were not available for Klebsiella spp. and E. faecium, so the breakpoints for E. coli and E. faecalis, as described above, were applied to these organisms.

Electronic patient charts were retrospectively analyzed to identify risk factors that could predispose patients to colonization with resistant bacteria. For those patients who were enrolled in the provincial drug benefit program, recent antibiotic use was retrieved from the drug profile viewer. Antibiotic history was not available for patients not registered in the provincial drug benefit program, but might have been available for patients with one or more previous hospital admissions within the specified study period. Data on exposure to fosfomycin within the previous 12 months were also collected when available.

\section{Outcomes}

The primary outcome of this study was the rate of susceptibility to fosfomycin of VRE and ESBL- or AmpC B-lactamaseproducing E. coli and Klebsiella spp. urinary isolates, as interpreted by the zone of inhibition. Isolates were classified as susceptible, intermediate, or resistant. According to the Clinical and Laboratory Standards Institute, "susceptible" indicates that standard antibiotic doses will inhibit the bacterial isolates with a high degree of clinical efficacy; "intermediate" implies that standard doses of antibiotic may achieve lower-than-normal inhibition, meaning that higher concentrations of antibiotic are required at the site of infection; and "resistant" suggests that the isolates are not inhibited by standard doses of antibiotic or that microbial resistance mechanisms are present (where clinical efficacy has not been reliably demonstrated by studies). ${ }^{14}$

The secondary outcome was the frequency of major risk factors associated with resistant uropathogens. The risk factors reviewed for patients with resistant Enterobacteriaceae were age 65 years or older, sex, recent (i.e., within the past 90 days) antibiotic use, recent (i.e., within the past 90 days) admission to hospital for $48 \mathrm{~h}$ or more, admission from a long-term care facility, international travel within the past 14 days, insertion of urinary catheter within the past 30 days, and history of malignancy. ${ }^{16,17}$ Similar risk factors were reviewed for patients with VRE, along with prolonged hospital stay ( $\geq 7$ days). ${ }^{18,19}$

\section{Statistical Analysis}

All data were analyzed through descriptive statistics. Antibiotic susceptibilities, expressed in percentages, were calculated as the proportion of susceptible urinary isolates relative to the total number of urinary isolates analyzed. Patient age was expressed as mean \pm standard deviation. All other risk factors were determined in terms of percentages.

\section{RESULTS}

\section{Outcomes}

A total of 106 ESBL- or AmpC B-lactamase-producing Enterobacteriaceae isolates (95 E. coli, 9 Klebsiella pneumoniae, and 2 Klebsiella oxytoca) and 31 isolates of vancomycin-resistant E. faecium were included in the analysis of fosfomycin susceptibility. Eleven urinary isolates of ESBL-producing E. coli and one of vancomycin-resistant $E$. faecium were excluded because of patient age ( $<18$ years) or repeat urine culture.

All 106 ESBL- and AmpC ß-lactamase-producing urinary isolates were susceptible to ertapenem (Table 1). Among the oral therapeutic options, fosfomycin had the highest susceptibility rate, at $96 \%$, whereas nitrofurantoin, trimethoprim-sulfamethoxazole, 
This single copy is for your personal, non-commercial use only.

For permission to reprint multiple copies or to order presentation-ready copies for distribution, contact CJHP at publications@cshp.ca

Table 1. Antibiotic Susceptibility of ESBL- and AmpC $\beta$-Lactamase-Producing Urinary Isolates

\begin{tabular}{|c|c|c|c|c|c|c|c|c|c|c|}
\hline \multirow{3}{*}{$\frac{\text { Susceptibility* }}{\text { E. coli }(n=95)}$} & \multicolumn{10}{|c|}{ Drug Tested; No. (\%) of Isolates } \\
\hline & Fosfomycin & Ertapenem & \multicolumn{2}{|c|}{ Nitrofurantoin } & \multicolumn{2}{|c|}{ Gentamicin } & \multicolumn{2}{|c|}{ TMP/SMX } & \multicolumn{2}{|c|}{ Ciprofloxacin } \\
\hline & & & & & & & & & & \\
\hline Susceptible & (96) & $95(100)$ & 79 & (83) & 68 & $(72)$ & 53 & $(56)$ & 13 & (14) \\
\hline Intermediate & $(1)$ & 0 & 9 & (10) & 0 & & 0 & & 0 & \\
\hline Resistant & (3) & 0 & 7 & (7) & 27 & $(28)$ & 42 & (44) & 82 & (86) \\
\hline \multicolumn{11}{|c|}{ Klebsiella spp. $(n=11)$} \\
\hline Susceptible & $11(100)$ & $11(100)$ & 3 & $(27)$ & 9 & $(82)$ & 2 & (18) & 5 & $(45)$ \\
\hline Intermediate & 0 & 0 & 6 & (55) & 0 & & 0 & & 1 & (9) \\
\hline Resistant & 0 & 0 & 2 & (18) & 2 & (18) & 9 & (82) & 5 & (45) \\
\hline
\end{tabular}

E. coli $=$ Escherichia coli, ESBL = extended-spectrum B-lactamase, TMP/SMX=trimethoprim-sulfamethoxazole.

*Susceptible = zone of inhibition $\geq 16 \mathrm{~mm}$ diameter; intermediate = zone of inhibition 13-15 mm diameter;

resistant = zone of inhibition $\leq 12 \mathrm{~mm}$ diameter.

Table 2. Antibiotic Susceptibility of Vancomycin-Resistant Enterococcus faecium Urinary Isolates

\begin{tabular}{|c|c|c|c|c|c|c|c|}
\hline \multirow{3}{*}{$\frac{\text { Susceptibility* }}{\text { Susceptible }}$} & \multicolumn{7}{|c|}{ Drug Tested; No. (\%) of Isolates $(n=31)$} \\
\hline & Fosfomycin & Linezolid & Tetracycline & Ampicillin & Penicillin & Nitrofurantoin & Ciprofloxacin \\
\hline & $25(81)$ & $31(100)$ & $21 \quad(68)$ & $2 \quad(6)$ & $1 \quad(3)$ & 0 & 0 \\
\hline Intermediate & $1 \quad(3)$ & 0 & 0 & 0 & 0 & $3(10)$ & 0 \\
\hline Resistant & $5(16)$ & 0 & $10 \quad(32)$ & (94) & $30(97)$ & $28(90)$ & $31(100)$ \\
\hline
\end{tabular}

*Susceptible = zone of inhibition $\geq 16 \mathrm{~mm}$ diameter; intermediate = zone of inhibition 13-15 mm diameter;

resistant $=$ zone of inhibition $\leq 12 \mathrm{~mm}$ diameter.

and ciprofloxacin had susceptibility rates of $83 \%, 56 \%$, and $14 \%$, respectively. The Klebsiella isolates also had a high susceptibility rate for fosfomycin $(100 \%)$, with high resistance rates for the typical first-line agents (Table 1). Among the 31 vancomycinresistant $E$. faecium urinary isolates, linezolid had the highest susceptibility (100\%), followed by fosfomycin (81\%) and tetracycline $(68 \%)$. Susceptibility to ampicillin, penicillin, nitrofurantoin, and ciprofloxacin was minimal (Table 2).

\section{Risk Factor Analysis}

Retrospective chart reviews for specific risk factors showed that patients harbouring these resistant organisms were often elderly, with mean ages of 70 years for patients with resistant Enterobacteriaceae urinary isolates and 72 years for those with vancomycin-resistant $E$. faecium urinary isolates. Patients with resistant isolates were more likely to be female and to have had antibiotic use within the past 90 days. Admission from long-term care facilities and international travel were not common risk factors. Patients with ESBL-producing organisms were often admitted with the resistant bacteria, whereas patients with vancomycin-resistant $E$. faecium were more likely to have acquired resistance though a prolonged hospital stay and/or recent hospital admissions (Table 3).

\section{Previous Exposure to Fosfomycin}

Five patients with ESBL infection and 2 patients with vancomycin-resistant $E$. faecium had been exposed to fosfomycin (as identified through drug profile viewer and inpatient records) before fosfomycin susceptibility testing. In one of the patients with vancomycin-resistant $E$. faecium, a single exposure to fosfomycin resulted in the development of resistance, with the zone of inhibition decreasing from $20 \mathrm{~mm}$ to $7 \mathrm{~mm}$ after exposure. However, in the remainder of these patients (including 2 patients who received 2 or more courses of fosfomycin treatment), susceptibility to fosfomycin was not affected by the prior exposure. Overall, previous exposure did not seem to affect fosfomycin susceptibility in the small number of patients tested in this study.

\section{DISCUSSION}

In this study, susceptibility rates were higher for fosfomycin than for other oral first-line antimicrobials for both resistant Enterobacteriaceae and vancomycin-resistant $E$. faecium urinary isolates. A recent systematic review examining the susceptibility of bacterial isolates to fosfomycin lacked information about VRE and Klebsiella susceptibilities in Canada. ${ }^{20}$ Two recently published Canadian studies reported fosfomycin susceptibilities ranging from $94.9 \%$ to $100 \%$ for ESBL- and AmpC ß-lactamase-producing E. coli. ${ }^{21,22}$ In the current study, the susceptibility rate of $96 \%$ falls within this previously reported range. In studies from other countries, ${ }^{23-25}$ susceptibility rates for Klebsiella spp. were slightly lower than those for E. coli; however, in the current study, all Klebsiella isolates were susceptible to fosfomycin. The 5 studies included in the systematic review of Vardakas and other ${ }^{20}$ reported fosfomycin susceptibilities for vancomycin-resistant E. faecium ranging from $30 \%$ to $100 \%$. 
This single copy is for your personal, non-commercial use only.

For permission to reprint multiple copies or to order presentation-ready copies for distribution, contact CJHP at publications@cshp.ca

\section{Table 3. Risk Factors Identified for Patients with Multidrug-Resistant Urinary Isolates}

\begin{tabular}{|c|c|c|c|c|}
\hline \multirow[b]{2}{*}{ Risk Factor } & \multicolumn{4}{|c|}{ Bacterial Isolate; No. (\%) of Patients* } \\
\hline & \multicolumn{2}{|c|}{$\begin{array}{c}\text { ESBL- or AmpC } \\
\text { B-Lactamase-Producing } \\
\text { Enterobacteriaceae }(n=106)\end{array}$} & \multicolumn{2}{|c|}{$\begin{array}{l}\text { Vancomycin-Resistant } \\
\text { Enterococcus faecium } \\
\qquad(n=31)\end{array}$} \\
\hline Age (years) (mean \pm SD) & \multicolumn{2}{|c|}{$70 \pm 19$} & \multicolumn{2}{|c|}{$72 \pm 12$} \\
\hline Sex, male & & $(38.7)$ & 10 & $(32.3)$ \\
\hline Recent antibiotic uset & 78 & $(83.0)$ & 29 & (93.5) \\
\hline Recent hospital admission‡ & 38 & $(35.8)$ & 20 & $(64.5)$ \\
\hline Admission from long-term c & are facility 13 & $(12.3)$ & 2 & $(6.5)$ \\
\hline International travel§ & 3 & $(2.8)$ & 0 & \\
\hline Urinary catheter & 29 & $(27.4)$ & 15 & $(48.4)$ \\
\hline History of malignancy & 31 & $(29.2)$ & 10 & (32.3) \\
\hline Hospital admission $\geq 7$ days & & NA & 16 & $(51.6)$ \\
\hline \multicolumn{5}{|c|}{$\begin{array}{l}\text { ESBL = extended-spectrum B-lactamase, NA = not applicable, SD = standard deviation. } \\
\text { *Except where indicated otherwise. } \\
\text { tIn the past } 90 \text { days. For this variable, data were available for } 94 \text { Enterobacteriaceae-positive } \\
\text { urinary isolates and all } 31 \text { E. faecium isolates. } \\
\text { tFor } 48 \text { h or longer in the past } 90 \text { days. } \\
\text { \$Within the past } 14 \text { days. } \\
\text { @Long-term indwelling catheter or urinary catheter inserted within the past } 30 \text { days. }\end{array}$} \\
\hline
\end{tabular}

According to the Infectious Diseases Society of America guidelines for acute uncomplicated cystitis, ${ }^{26}$ trimethoprimsulfamethoxazole should no longer be recommended for empirical treatment where local resistance rates exceed $20 \%$. As well, fluoroquinolones are not considered an appropriate choice for patients in the community if resistance rates are above $10 \%{ }^{26}$ Clinicians have applied this concept of a resistance threshold (typically 10\%-20\%) to other antimicrobials when selecting empiric therapy for patients. In the current study, only ertapenem, linezolid, and fosfomycin consistently had resistance rates below $20 \%$, and thus would be considered reliable empiric therapies. As indicated by the provincial drug benefit formulary, the acquisition cost of ertapenem and linezolid can be 30 times more than that of fosfomycin per course of treatment for uncomplicated lower UTI. It is clear that broad-spectrum antibiotics are more costly than fosfomycin and do not demonstrate significantly greater susceptibility rates. Nagel and others ${ }^{27}$ compared the clinical efficacy and economic impact of fosfomycin and other antibiotic therapies for treatment of lower UTIs due to ESBL-producing Enterobacteriaceae and VRE. They showed that the average length of treatment was lower among those receiving fosfomycin (2.93 versus 7.19 days), and the mean antibiotic cost per patient was also lower (US\$106.74 versus US\$269.55), with similar efficacy. In the fosfomycin group, $81 \%$ of patients received a single oral dose of 3 g. ${ }^{27}$ Another study comparing fosfomycin with ertapenem for the treatment of lower UTIs due to ESBL-producing organisms further confirmed that total duration of antibiotic treatment, including outpatient treatment days, was significantly longer for the ertapenem group than the fosfomycin group. ${ }^{28}$

The analysis presented here suggests that the frequency of several risk factors was greater among patients with VRE. VRE was more likely to be acquired through hospitalization, especially if the hospital stay was prolonged, which reflects the ongoing issue of infection control and transmission in the health care setting. A larger sample size would be required to fully assess the impact of this factor. In addition, a higher percentage of patients with VRE had urinary catheterization, but this could be attributable to prolonged hospitalization.

Although fosfomycin is not a new antibiotic, its use has been infrequent, especially in Canada, because of difficulty in acquiring the drug in previous years and limited knowledge about its local susceptibility rates. There is concern that increased use of this drug is associated with increased resistance. ${ }^{29,30}$ In the current study, a small number of patients $(n=7)$ had exposure to fosfomycin before susceptibility testing. In one of these patients, in vitro resistance developed after the exposure. Although there have been studies indicating development of in vitro resistance with increases in use, resistance was found to be rare in areas where fosfomycin was widely used in clinical practice. ${ }^{29-32}$ Low resistance rates may be due to the achievement of high concentrations in the urine and greater adherence with single-dose therapies. ${ }^{32}$

To our knowledge, this study is the first in Canada to test fosfomycin susceptibility against several multidrug-resistant uropathogens from patients with UTIs, including E. coli, Klebsiella spp., and vancomycin-resistant $E$. faecium. Susceptibilities for typical first-line agents were analyzed to establish any significant differences in susceptibility rates among these agents. The results confirmed fosfomycin as the most reliable oral option for treating infections due to multidrug-resistant uropathogens. This study also examined the frequency of major risk factors associated with these resistant uropathogens.

One limitation of this study was the limited assessment of clinical outcomes, despite utilization of fosfomycin by many of the patients whose urinary isolates were included in the study. 
Since fosfomycin susceptibility breakpoints for organisms other than E. coli and E. faecalis have not been established by the Clinical and Laboratory Standards Institute, the breakpoints for these 2 organisms were applied to susceptibility testing for Klebsiella spp. and E. faecium. Despite this limitation, many studies have employed the same method for fosfomycin susceptibility testing. ${ }^{23-25,33,34}$ Recent antibiotic use was captured for the majority of the patients, and previous exposures to fosfomycin were minimal. This result may be due to practitioners' lack of awareness of the commercial availability of fosfomycin. Thus, this study may generate increased awareness about the potential use of fosfomycin.

\section{CONCLUSION}

Favourable susceptibility rates make fosfomycin the most reliable oral option for the treatment of lower UTIs in patients with suspected or documented multidrug-resistant uropathogens. The results of this study may influence practitioners' approaches to choosing antibiotics for the treatment of UTIs caused by multidrug-resistant Enterobacteriaceae and VRE. A change in approach could, in turn, lead to a reduction in the selection of more resistant organisms through a decrease in the use of broadspectrum antibiotics. Single-dose oral therapy for uncomplicated UTIs increases cost savings and, more importantly, may improve patient satisfaction and the quality of patient care by reducing invasive procedures.

\section{References}

1. Monurol (fosfomycin tromethamine) [product monograph]. Saint-Laurent (QC): Paladin Labs Inc; 2016 Aug 17.

2. Qiao LD, Zheng B, Chen S, Yang Y, Zhang K, Guo HF, et al. Evaluation of three-dose fosfomycin tromethamine in the treatment of patients with urinary tract infections: an uncontrolled, open-label, multicenter study. BMJ Open. 2013;3(12):e004157.

3. Neuner EA, Sekeres J, Hall GS, van Duin D. Experience with fosfomycin for treatment of urinary tract infections due to multidrug-resistant organisms. Antimicrob Agents Chemother. 2012;56(11):5744-8.

4. Senol S, Tasbakan M, Pullukcu H, Sipahi OR, Sipahi H, Yamazhan T, et al. Carbapenem versus fosfomycin tromethanol in the treatment of extendedspectrum beta-lactamase-producing Escherichia coli-related complicated lower urinary tract infection. J Chemother. 2010;22(5):3557.

5. Falagas ME, Kastoris AC, Kapaskelis AM, Karageorgopoulos DE. Fosfomycin for the treatment of multidrug-resistant, including extendedspectrum B-lactamase producing, Enterobacteriaceae infections: a systematic review. Lancet Infect Dis. 2010;10(1):43-50.

6. Lob SH, Nicolle LE, Hoban DJ, Kazmierczak KM, Badal RE, Sahm DF. Susceptibility patterns and ESBL rates of Escherichia coli from urinary tract infections in Canada and the United States, SMART 2010-2014. Diagn Microbiol Infect Dis. 2016;85(4):459-65.

7. Falagas ME, Karageorgopoulos DE. Extended-spectrum B-lactamaseproducing organisms. J Hosp Infect. 2009;73(4):345-54.

8. Wilson DT, May DB. Potential role of fosfomycin in the treatment of community-acquired lower urinary tract infections caused by extended-spectrum B-lactamase-producing Escherichia coli. Am J Ther. 2013;20(6):685-90.

9. Reffert JL, Smith WJ. Fosfomycin for the treatment of resistant gram-negative bacterial infections. Pharmacotherapy. 2014;34(8):845-57.

10. McLaughlin M, Advincula MR, Malczynski M, Qi C, Bolon M, Scheetz $\mathrm{MH}$. Correlations of antibiotic use and carbapenem resistance in Enterobacteriaceae. Antimicrob Agents Chemother. 2013;57(10):5131-3.
11. Heintz BH, Halilovic J, Christensen CL. Vancomycin-resistant enterococcal urinary tract infections. Pharmacotherapy. 2010;30(11):1136-49.

12. Falagas ME, Vouloumanou EK, Togias AG, Karadima M, Kapaskelis AM, Rafailidis PI, et al. Fosfomycin versus other antibiotics for the treatment of cystitis: a meta-analysis of randomized controlled trials. J Antimicrob Chemother. 2010;65(9):1862-77.

13. Michalopoulos AS, Livaditis IG, Gougoutas V. The revival of fosfomycin. Int J Infect Dis. 2011;15(11):e732-9.

14. M100s: Performance standards for antimicrobial susceptibility testing. 26th ed. Wayne (PA): Clinical and Laboratory Standards Institute; 2016.

15. Reller LB, Weinstein M, Jorgensen JH, Ferraro MJ. Antimicrobial susceptibility testing: a review of general principles and contemporary practices. Clin Infect Dis. 2009;49(11):1749-55.

16. Ben-Ami R, Rodríguez-Baño J, Arslan H, Pitout JDD, Quentin C, Calbo ES, et al. A multinational survey of risk factors for infection with extendedspectrum B-lactamase-producing Enterobacteriaceae in nonhospitalized patients. Clin Infect Dis. 2009;49(5):682-90.

17. Rodríguez-Baño J, Alcalá JC, Cisneros JM, Grill F, Oliver A, Horcajada JP, et al. Community infections caused by extended-spectrum beta-lactamaseproducing Escherichia coli. Arch Intern Med. 2008;168(17):1897-1902.

18. Karki S, Houston L, Land G, Bass P, Kehoe R, Borrell S, et al. Prevalence and risk factors for VRE colonisation in a tertiary hospital in Melbourne, Australia: a cross sectional study. Antimicrob Resist Infect Control. 2012;1:31.

19. Tornieporth NG, Roberts RB, John J, Hafner A, Riley LW. Risk factors associated with vancomycin-resistant Enterococcus faecium infection or colonization in 145 matched case patients and control patients. Clin Infect Dis. 1996;23(4):767-72.

20. Vardakas KZ, Legakis NJ, Triarides N, Falagas ME. Susceptibility of contemporary isolates to fosfomycin: a systematic review of literature. Int J Antimicrob Agents. 2016;47(4):269-85.

21. Beuk C, Hill C, Whitehead S, Blondel-Hill E, Wagner K, Cheeptham N. Determination of susceptibility to fosfomycin and tigecycline of Enterobacteriaceae, particularly Escherichia coli isolates, producing extended-spectrum ß-lactamases from multiple regional Canadian hospitals. Can J Infect Dis Med Microbiol. 2013;24(3):e80-2.

22. Karlowsky JA, Denisuik AJ, Lagacé-Wiens PRS, Adam HJ, Baxter MR, Hoban DJ, et al. In vitro activity of fosfomycin against Escherichia coli isolated from patients with urinary tract infections in Canada as part of the CANWARD surveillance study. Antimicrob Agents Chemother. 2014;58(2): 1252-6.

23. Demir T, Buyukguclu T. Evaluation of the in vitro activity of fosfomycin tromethamine against gram-negative bacterial strains recovered from community- and hospital-acquired urinary tract infections in Turkey. Int J Infect Dis. 2013;17(11):e966-70.

24. Nakamura T, Komatsu M, Yamasaki K, Fukuda S, Higuchi T, Ono T, et al. Susceptibility of various oral antibacterial agents against extended spectrum B-lactamase producing Escherichia coli and Klebsiella pneumoniae. I Infect Chemother. 2014;20(1):48-51.

25. Liu HY, Lin HC, Lin YC, Yu SH, Wu WH, Lee YJ. Antimicrobial susceptibilities of urinary extended-spectrum beta-lactamase-producing Escherichia coli and Klebsiella pneumoniae to fosfomycin and nitrofurantoin in a teaching hospital in Taiwan. J Microbiol Immunol Infect. 2011;44(5): 364-8.

26. Gupta K, Hooton TM, Naber KG, Wullt B, Colgan R, Miller LG. International clinical practice guidelines for the treatment of acute uncomplicated cystitis and pyelonephritis in women: a 2010 update by the Infectious Disease Society of America and the European Society for Microbiology and Infectious Diseases. Clin Infect Dis. 2011;52(5):e103-20.

27. Nagel JL, Washer L, Kunapuli A, Heidmann J, Pisani J, Gandhi T. Clinical efficacy of fosfomycin for the treatment of complicated lower tract and uncomplicated urinary tract infections. Int Arch Med. 2015;8(151):1-7.

28. Veve MP, Wagner JL, Kenney RM, Grunwald JL, Davis SL. Comparison of fosfomycin to ertapenem for outpatient or step-down therapy of extendedspectrum B-lactamase urinary tract infections. Int J Antimicrob Agents. 2016;48(1):56-60.

29. Oteo J, Orden B, Bautista V, Cuevas O, Arroyo M, Martínez-Ruiz R, et al. CTX-M-15-producing urinary Escherichia coli O25b-ST131-phylogroup 
B2 has acquired resistance to fosfomycin. J Antimicrob Chemother. 2009; 64(4):712-7.

30. Oteo J, Bautista V, Lara N, Cuevas O, Arroyo M, Fernández S, et al. Parallel increase in community use of fosfomycin and resistance to fosfomycin in extended-spectrum ß-lactamase (ESBL)-producing Escherichia coli. J Antimicrob Chemother. 2010;65(11):2459-63.

31. Sastry S, Clarke LG, Alrowais H, Querry AM, Shutt KA, Doi Y. Clinical appraisal of fosfomycin in the era of antimicrobial resistance. Antimicrob Agents Chemother. 2015;59(12):7355-61.

32. Zhanel GG, Walkty AJ, Karlowsky JA. Fosfomycin: a first-line oral therapy for acute uncomplicated cystitis. Can J Infect Dis Med Microbiol. 2016;2016: 2082693.

33. Maraki S, Samonis G, Rafailidis PI, Vouloumanou EK, Mavromanolakis E, Falagas ME. Susceptibility of urinary tract bacteria to fosfomycin. Antimicrob Agents Chemother. 2009;53(10):4508-10.

34. Hirsch EB, Raux BR, Zucchi PC, Kim Y, McCoy C, Kirby JE, et al. Activity of fosfomycin and comparison of several susceptibility testing methods against contemporary urine isolates. Int J Antimicrob Agents. 2015;46(6):642-7.
Linda B Ou, BSc(Pharm), ACPR, was, at the time of this study, a Pharmacy Resident at Windsor Regional Hospital, Windsor, Ontario. She is now a candidate in the Master of Science Epidemiology program at McGill University, Montréal, Quebec.

Lynn Nadeau, PharmD, is a Clinical Pharmacy Specialist in Infectious Diseases at Windsor Regional Hospital, Windsor, Ontario.

Competing interests: None declared.

Address correspondence to:

Dr Lynn Nadeau

Windsor Regional Hospital - Ouellette Campus

1030 Ouellete Avenue

Windsor ON N9A 1E1

e-mail: Lynn.Nadeau@wrh.on.ca

Funding: Financial support for this study was provided by the Department of Pharmacy, Windsor Regional Hospital.

Acknowledgements: The authors would like to thank the staff at Integrated Hospital Laboratories Service, Windsor-Essex, for assistance with data collection and Dr David Edwards, University of Waterloo, for statistical support.

\section{cshp 在 scph}

\section{CSHP Members Save on}

\section{ASHP and Pharmaceutical Press Publications!}

\section{CSHP members are invited to place their orders for all ASHP and Pharmaceutical Press

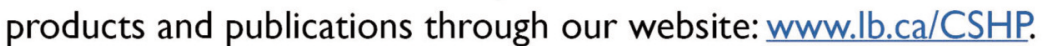 \\ CSHP members also save on cross border shipping charges through Login Canada!}

CSHP members can obtain the password needed to place an order for ASHP, Pharmaceutical Press, and other products and publications by going to the Products tab on the CSHP homepage. From the drop down menu select ASHP and Pharmaceutical Press Publications. Members will be asked to login before being allowed to proceed to CSHP's virtual bookstore.

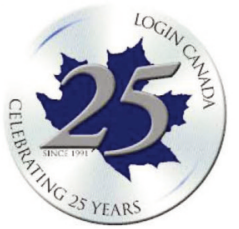

